\title{
Research Paper: Reliability of the Modified In-hand Manipulation Test and the Relationship Between In-hand Manipulation and Handwriting
}

\author{
Marie-Laure Kaiser $^{1 *}$ (D), Caroline André Carrascob² (i) \\ 1. Departemant of Direction, School of Health Sciences, University of Applied Sciences and Arts Western Switzerland, Genève, Switzerland. \\ 2. Departemant of Occupational Therapy Service, University Hospital, Pierre-Decker, Lausanne, Switzerland.
}

\begin{tabular}{l|l}
\hline $\begin{array}{l}\text { use your device to san } \\
\text { and read the article online }\end{array}$ \\
Cftation: Kaiser ML, Carrascob CA. Reliability of the Modified In-hand Manipulation Test and the Relationship Between In-hand \\
Manipulation and Handwriting. Iranian Rehabilitation Journal. 2019; 17(3):279-284. http://dx.doi.org/10.32598/irj.17.3.279
\end{tabular}

Article info:

Received: 17 Nov 2018

Accepted: 03 Mar 2019

Available Online: 01 Sep 2019

Keywords:

Children, Motor skills, Child

\section{A B ST RACT}

Objectives: This study aimed at analyzing the test-retest and interrater reliabilities of the Test of In-Hand Manipulation-Modified (TIHM-M), as well as the relationship between in-hand manipulation and handwriting.

Methods: We administered the TIHM-M twice, two days apart, and the Beknopte Beoordelingsmethode voor Kinder Handschriften (BHK) - Concise Assessment Method for children's handwriting test once to 105 children (51 boys and 54 girls) aged 6.5 to 9.5 years in regular school. Speed and quality of in-hand manipulation and handwriting were recorded. We performed a Pearson correlation test for the relationship between the speeds and the qualities of the TIHM-M and the BHK.

Results: Test-retest of the TIHM-M was excellent for the speed (ICC $=0.84 ; 95 \%$ CI:0.730.90 ) and good for the quality (ICC $=0.62,95 \% \mathrm{CI}: 0.36-0.78)$. Interrater reliabilities for both speed (ICC $=0.87 ; 95 \%$ CI:0.78-0.92) and quality (ICC $=0.86,95 \% \mathrm{CI}: 0.60-0.84)$ were also excellent. We found a significant relationship between the speeds of the TIHM-M and the $\mathrm{BHK}$, but there was no significant relationship between the qualities of handwriting and the TIMH-M.

Discussion: The TIMH-M assessed the speed and the quality of in-hand manipulations, and it had good reliability. Nevertheless, the relationship between in-hand manipulation and handwriting needs more investigation to be confirmed.

\section{* Corresponding Author:}




\section{Highlights}

- In-hand manipulations are part of fine motor skills and play a role in activities of daily living.

- The modified in-hand manipulation test that provides a total score based on speed and quality performance is a reliable test for children aged 6.5 to 9.5 years old.

- The relationship between in-hand manipulation and handwriting needs more investigation to be confirmed.

\section{Plain Language Summary}

In-hand manipulations are movements that are done with one hand to manipulate small objects. They are part of fine motor skills and play a role in activities such as using a key, buttoning, manipulations of coins, or using fork and knife. It is essential to have a reliable test to assess these manipulations. We used a test that includes rotation of 5 pegs, translation of 3, 4, and 5 pegs from fingers to palm and reverse, and shift that are finger movements along with a stick forth and back. The objectives were to analyze: 1 . the test-retest reliability to know if the results of the test are similar after a short period of time; 2. the interrater reliability to identify if two assessors will find the same results; 3 . the relationship between the in-hand manipulation and handwriting to understand the role of in-hand manipulation in handwriting. We found a good test-retest and interrater reliabilities, which means that this test can be used by professionals to identify children with in-hand difficulties. A significant correlation was established between the speed of the TIHM-M and the speed of handwriting, which means if the children are slow to realize in-hand manipulation, they may be slow in handwriting, too. More investigation is needed to confirm this point.

\section{Introduction}

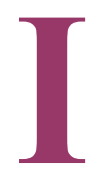

n-hand manipulations are coordinated movements of the fingers of one hand to adjust an object in that hand before using or releasing it [1]. Three types of movements are identified: rotation, translation from fingers to palm and from palm to fingers, and shift; the latter is an adjustment of fingers to adjust the grip on the objects. In-hand manipulation is important in daily living activities, such as using a spoon, manipulating coins, orienting, and positioning a key to enter it in a lock, or coloring and or handwriting [2].

There are two categories of standardized assessment of in-hand manipulation. The first category is composed of items with objects of daily living [3], and the second one uses a pegboard and pegs $[4,5]$. In the first category, we find the "Observation Protocol on In-hand Manipulation" [3]. It contains 60 items realized with objects of daily living such as cubes or dowels. It measures the quality and speed of in-hand manipulation. The value of each item goes from 0 to 4 , and the total score goes from 0 to 180 . Breslin and Exner described fair discrimination validity. The other test is "Functional Skill Development" [6]. It contains 8 items, using spoon, pencil, or buttons. The score for each item goes from zero to five and a total score between zero and 40 .
In the second category, the Test of In-Hand Manipulation (TIHM) was developed by Case-Smith [4]. It requires a $180^{\circ}$ rotation of 5 pegs and translation of 2 pegs. The number of drops was registered. Pehoski et al. included two types of rotation in the TIHM [7]; one required returning 5 pegs upside down, whereas the second required turning the same pegs 10 times between the digits. They recorded speed and quality based on different strategies of manipulation, but they did not provide standardized scores. The test has not been validated.

Pont et al. used a modified version of this test (TIHMR) with a group of children aged 5.5 to 6.5 years. The results of translations with four and five pegs were considered, whereas translation of two and three pegs was practice task [8]. The following criteria were retained to assess the items: 1. speed; 2 . number of dropped peg; 3 . stabilization; and 4. quality of in-hand manipulation. The latter was abandoned because of the lack of construct validity. High interrater reliability and weak test-retest reliability were found.

The relationship between in-hand manipulation and pre-graphism skills was analyzed in the studies of Humphries, Jewell, Rosenberger, and Case-Smith [4, 6]. They found a strong and moderate correlation, respectively. The relationship between in-hand manipulation and handwriting was analyzed by Cornhill and Case- 
Smith, who found a strong correlation between the speed of the rotation and translation of pegs and the legibility of handwriting in school-aged children with a mean age of 7.1 years [9]. Brown and Link also found a significant correlation between the speeds of in-hand manipulation and handwriting in school-aged children with a mean age of 7.2 years [10]. If significant correlations were found, the predictive value of in-hand manipulation was never found alone, but it was always associated with another components. In the Cornhill and Case-Smith's study, the association of visual-motor integration and in-hand manipulation was a predictive value of the legibility of handwriting, whereas in a study carried out by Brown and Link, the association of visual-closure skills and in-hand manipulation was predictive of the speed of handwriting $[9,10]$.

In summary, the assessments of in-hand manipulation lack clear reliability values and mostly overlook the quality. Furthermore, no clear relationship has been found between in-hand manipulation and handwriting. Therefore, we decided to conduct a study to validate an in-hand manipulation test in children aged 6.5 to 9.5 years to analyze interrater reliability and test-retest reliability, as well as the relationship between in-hand manipulation and handwriting and the predictive value of in-hand manipulation on handwriting.

\section{Methods}

\section{Study participants}

The children were recruited from 4 regular schools in the French part of Switzerland. They were recruited by convenience sampling method. We included 105 children (54 girls and 51 boys) from different socioeconomic backgrounds. In Switzerland, the children mostly follow regular classes, and children in a given class may come from different socio-cultural backgrounds. The children were classified into three age groups: 1 . Thirty seven children (17 girls and 20 boys) aged 6.5 to 7.4 years (Mean \pm SD:7.00 \pm 0.29 y); 2 . Forty one children (21 girls and 20 boys) aged 7.5 to 8.4 years (Mean \pm SD:7.87 \pm 0.27 $\mathrm{y})$; and 3 . Twenty seven children (16 girls and 11 boys) aged 8.5 to 9.5 years $(M e a n \pm S D: 8.91 \pm 0.37$ y). There were 10 left-handed children, 4 in the first two groups and 2 in the last group. The inclusion criteria were being in the grade level according to age and written informed consent of the parents.

The exclusion criterion was no known physical or developmental disorder based on the information of the parents and / or the teachers.

\section{Instrumentation}

We used a wooden pegboard of the 9-hole test, measuring $12.7 \times 12.7 \times 4.5 \mathrm{~cm}$. The depth of the holes was 2 $\mathrm{cm}$. The distance between the holes was $3.5 \mathrm{~cm}$ (heart to heart). The pegboard was placed on a non-slip pad $(38 \times 29 \mathrm{~cm})$. The pegs were $3.8 \mathrm{~cm}$ long and $0.6 \mathrm{~cm}$ in diameter, with a drawing of a face on one side and of feet on the other. For the assessment of shift, we used a stick of the same diameter and a length of $17 \mathrm{~cm}$ and $0.6 \mathrm{~cm}$ in diameter with a line at $2 \mathrm{~cm}$ from each end.

We adapted the protocol described in the study of Pont et al. [8]. Firstly, we added an item to assess shift because this movement is essential to adjust the pencil after picking it up. Secondly, rather than having two trials of each item, we proposed as in the Movement $\mathrm{ABC}$, an informal and untimed trial and, then, twotimed and formal trials [11].

The children executed 5 different tasks: 1 . Rotation of five pegs; 2 . Translation of three pegs; 3 . Translation of four pegs; 4 . Translation of five pegs; and 5 . Shift.

We used the same criteria for the quality of rotation and translation as Pont et al. (2008), i.e. the number of drop and stabilization of a peg on an external surface. If the child dropped a peg outside of the pad, the item would be failed. Each error can be counted only once for one peg. For example, if a child stabilized a peg and further dropped it, then, only the drop would be counted. For the translation items, we added a third criterion, i.e. no contact of the peg with the thumb, which means that the child did not use the thumb to translate the peg from the palm to the fingers to drop it into the hole.

For the shift, a failure would be considered if the child dropped the stick or while moving, the stick had an angle of $\geq 45^{\circ}$ on more than half of the distance, i.e. using gravity for moving the stick. For example, the child holds the peg between fingers. A trial would also be a failure if the grip position of the fingers did not reach the marked line at the end of the stick with a tolerance of $1 \mathrm{~cm}$.

For rotation, the raw score of the quality could range between 0 and 5 . For the translation of 3, 4, or 5 pegs, it was 0 to 3,0 to 4 , and 0 to 5 , respectively; and for the raw score of shift, it was between 0 and 2 . The total score of quality can be between 0 and 19 .

A total score for the speed was calculated, using the best speed of each item. For the quality, the raw score of 
each item corresponding to the best speed was added to obtain the total quality score.

We used the French version of the BHK-Concise Assessment Method for children's handwriting test [12]. It requires copying a text in 5 minutes. It is targeted at children from kindergarten to the sixth grade. The total score for the quality ranges between 0 and 65 . The total score for the speed is the number of letters written during 5 minutes.

\section{Procedure}

Two occupational therapists (authors) and 5 Occupational Therapy students (OT students), trained by the second author, administered the TIHM-M. The training included a presentation of the test and a simulation of the administration. Then, the students administered the TIHM-M to two children, which was video-recorded. The second author gave feedback to the students. The authors of the article wrote a standardized instruction for the administration and the scoring.

The TIHM-M was administered at school, usually with the assessor and the child alone in a small room, but sometimes with two assessor-child pairs in a larger room. Two days later, the test was administered for the second time. This short interval prevented a retest effect. Each session was video-recorded. Two authors and / or a research assistant analyzed the videos and coded the speed and the quality for the interrater reliability. The French version of the Beknopte Beoordelingsmethode voor Kinder Handschriften (BHK)Concise Assessment Method for children's handwriting test was administered during the first or the second assessment of the TIHM-M [12].

\section{Data analysis}

We performed an intra-class correlation to analyze the interrater reliability [13]. As the raters were not similar for all children, we applied a 1-way random effect, using SPSS V. 23.

For test-retest, we performed an intra-class correlation (two-mixed model). Fleiss considered a score above 0.75 as excellent, a score between 0.60 and 0.74 as good, a score between 0.40 and 0.59 as a medium, and a score under 0.40 as poor [14]. A Pearson correlation test was used to analyze the relationship between in-hand manipulation and handwriting and stepwise regression for the predictive value of the TIMH-M on the BHK.

\section{Results}

For the TIHM-M, we found excellent intra-class correlations between inter-raters for both speed ( $\mathrm{ICC}=0.85 ; 95 \%$ CI:0.75-0.91) and quality (ICC $=0.87 ; 95 \% \mathrm{CI}: 0.78-0.92$ ). For the test-retest, the ICC was excellent for the speed ( $\mathrm{ICC}=0.84 ; 95 \% \mathrm{CI}=0.73-0.90$ ) and good for the quality (ICC $=0.62,95 \% \mathrm{CI}=0.36-0.78)$ (Table 1 and 2 ).

Table 1. Interraters reliability ( $\mathrm{n}=53)$, mean, standard deviation, intraclass correlation, confidence of interval

\begin{tabular}{ccccc}
\hline Total score & Raters & Mean \pm SD & ICC & $95 \% \mathbf{C l}$ \\
\hline Speed & 1 & $47.94 \pm 9.25$ & 0.85 & $0.75-0.91$ \\
& 2 & $46.22 \pm 10.25$ & & $0.78-0.92$ \\
Quality & 1 & $3.15 \pm 2.59$ & 0.87 & \\
& 2 & $2.79 \pm 2.69$ & & \\
\hline
\end{tabular}

Iranian Rehabilitation Journa

Table 2. Test-retest reliability $(\mathrm{n}=56)$, mean, standard deviation, intraclass correlation, confidence of interval

\begin{tabular}{ccccc}
\hline Total score & Test-Retest & Mean \pm SD & ICC & $95 \% \mathrm{Cl}$ \\
\hline \multirow{2}{*}{ Speed } & Test & $50 \pm 10.24$ & $0.73-0.90$ \\
& Retest & $48 \pm 10.18$ & & 0.84 \\
Quality & Test & $2.76 \pm 3.05$ & 0.62 & $0.36-0.78$ \\
& Retest & $2.21 \pm 2.39$ & & \\
\hline
\end{tabular}


The Pearson correlation between the total speed of the TIHM-M and the speed of the BHK $(r=0.21 ; \mathrm{P}<0.05)$ and between the total speed of the TIMH-M and the quality of the $\mathrm{BHK}(\mathrm{r}=0.20 ; \mathrm{P}<0.05)$ was weak but significant. No significant correlation was found between the quality of handwriting and the quality of the TIHMM. No predictive value of the TIMH-M was found, too.

\section{Discussion}

We found a good test-retest reliability for speed and quality, whereas Pont et al. obtained a weak test-retest reliability of the TIHM-R. Our re-test interval was two days, whereas theirs was two weeks [8]. The children in their study may have practiced or developed their skills for two weeks. Furthermore, they were also younger, and their production may have been less stable. Finally, our test differs from the one of Pont et al. study, because we added other criteria of quality and the shift item. These different factors could explain the difference in results.

We found a good interrater reliability for speed and quality. These results are similar to those of Pont et al. study, who analyzed the interrater reliability for the speed and two criteria of quality [15]. We added two more criteria measuring the quality and still found a good reliability.

We found a significant relationship between the speeds of in-hand manipulation and handwriting, whereas Feder et al. did not, they only found a significant correlation in a sample of premature children of 6 to 7 years old and at risk for developmental disorders [16]. This difference of results may be due to the different handwriting tests. We used a single near-point copying test, whereas Feder et al. administered the Evaluation Tool of Children's Handwriting-Manuscript that assessed handwriting in 6 different tasks of the alphabet, numeral writing, nearpoint, and far-point copying, composition, and dictation [17]. The broader assessment may better identify the handwriting difficulties of the children.

Based on our results, we cannot conclude that the TIHM-M is valid to identify children with handwriting difficulties. We did not find any predictive value because there were probably many endogenous and exogenous factors that influenced the speed and the quality of handwriting [18].

As the TIHM-M provides a total score, including quality and speed, it can better identify children, who have difficulties to perform a fine motor task than a test that only assesses the speed of in-hand manipulation. While performing a test, some children may choose the speed, but not the quality, for example, they may drop or stabilize pegs. By not considering both speed and quality performances, we can miss some children with in-hand manipulation problems.

Despite good reliability of TIHM-M, it still has some limitations due to the limited sample, the lack of construct, and external validities. A replication of our study with a larger sample should be done to confirm our results. Research on the construct validity should be conducted to analyze whether the TIHM-M discriminates between children with and without fine motor skills. We used a unique handwriting copy task that did not reflect all the handwriting tasks at school. Our study should be reproduced, using a handwriting assessment with several tasks and a larger sample to check for proper external validity.

\section{Conclusions}

TIHM-M is a reliable test. The relationship between the TIHM-M and handwriting is not strong, and when children have inadequate handwriting, intrinsic or extrinsic factors should be assessed to find the relevant factors related to this handwriting $[18,19]$.

\section{Ethical Considerations}

\section{Compliance with ethical guidelines}

The Ethics Committee of the Canton of Vaud in Switzerland approved the research project in 2012. The parents were informed of the research protocol and provided their written consent. They could withdraw their child from the research at any time.

\section{Funding}

This research did not receive any specific grant from funding agencies in the public, commercial, or not-forprofit sectors.

\section{Authors' Contributions}

Methodology, investigation, resources, data curation, review \& editing, visualization: Marie-Laure Kaiser, Caroline André Carrascob; Software, validation, formal analysis, writing-original draft preparation, writing, supervision: Marie-Laure Kaiser; Project administration, funding acquisition: Caroline André Carrascob.

\section{Conflict of interest}

The Authors decleared no conflict of interest 


\section{References}

[1] Exner CE. In-hand manipulation skills. In: Case-Smith J Pehoski C, editors. Development of Hands Skills in the Child. Rockville, Maryland: American Occupational Therapy Association; 1992.

[2] Humphries T, Snider L, McDougall B. Clinical evaluation of the effectiveness of sensory integrative and perceptual motor therapy in improving sensory integrative function in children with learning disabilities. The Occupational Therapy Journal of Research. 1993; 13(3):183-97. [DOI:10.1177/1539449293013 00302]

[3] Breslin D, Exner CE. Construct validity of the in-hand manipulation test: A discriminant analysis with children without disability and children with spastic diplegia. American Journal of Occupational Therapy. 1999; 53(4):381-6. [DOI:10.5014/ ajot.53.4.381] [PMID]

[4] Case-Smith J. Clinical interpretation of development of inhand manipulation and relationship with activities. American Journal of Occupational Therapy. 1995; 49(8):772-4. [DOI:10.5014/ajot.49.8.772] [PMID]

[5] Pehoski C, Henderson A, Tickle-Degnen L. In-hand manipulation in young children: Rotation of an object in the fingers. American Journal of Occupational Therapy. 1997; 51(7):54452. [DOI:10.5014/ajot.51.7.544] [PMID]

[6] Humphry R, Jewell K, Rosenberger RC. Development of inhand manipulation and relationship with activities. American Journal of Occupational Therapy. 1995; 49(8):763-71. [DOI:10.5014/ajot.49.8.763] [PMID]

[7] Pehoski C, Henderson A, Tickle-Degnen L. In-hand manipulation in young children: Translation movements. The American Journal of Occupational Therapy. 1997; 51(9):719-28. [DOI:10.5014/ajot.51.9.719] [PMID]

[8] Pont K, Wallen M, Bundy A, Case-Smith J. Reliability and validity of the Test of In-Hand Manipulation in children ages 5 to 6 years. The American Journal of Occupational Therapy. 2008; 62(4):384-92. [DOI:10.5014/ajot.62.4.384] [PMID]

[9] Cornhill H, Case-Smith J. Factors that relate good and poor handwriting. The American Journal of Occupational Therapy. 1996; 50(9):732-9. [DOI:10.5014/ajot.50.9.732] [PMID]

[10] Brown T, Link J. The association between measures of visual perception, visual-motor integration, and in-hand manipulation skills of school-age children and their manuscript handwriting speed. British Journal of Occupational Therapy. 2016; 79(3):163-71. [DOI:10.1177/0308022615600179]

[11] Henderson SE, Sugden DA, Barnett A. Movement assessment battery for children- $2^{\text {nd }}$ edition (Movement ABC-2) Dutch version supervised by Smits-Engelsman. London: Pearson; 2010.

[12] Charles M, Soppelsa R, Albaret JM. [BHK- rapid evaluation scale of writing in children (French)]. Paris: ECPA; 2003.

[13] Landers RN. Computing Intraclass Correlations (ICC) as estimates of interrater reliability in SPSS. The Winnower [Internet]. [Updated 2015 ???] Available from: https://thewinnower.com/papers/1113-computing-intraclass-correlationsicc-as-estimates-of-interrater-reliability-in-spss).
[14] Fleiss JL. The measurement of interrater agreement. In Fleiss JL, editor. Statistical Methods for Rates and Proportion. New York: Wiley; 1981

[15] Adolph KE, Cole WG, Komati M, Garciaguirre JS, Badaly D, Lingeman JM, et al. How do you learn to walk? Thousands of steps and dozens of falls per day. Psychological Science. 2012; 23(11):1387-94. [DOI:10.1177/0956797612446346] [PMID] [PMCID]

[16] Feder KP, Majnemer A, Bourbonnais D, Platt R, Blayney $\mathrm{M}$, Synnes A. Handwriting performance in preterm children compared with term peers at age 6 to 7 years. Developmental Medicine \& Child Neurology. 2005; 47(3):163-70. [DOI:10.1017/S0012162205000307]

[17] Amundson SJ. Evalution tool of children's handwriting: ETCH examiner's manual. Homer, Alaska: AK: O.T. KIDS; 1995

[18] Kaiser ML, Albaret JM, Doudin PA. Relationship between visual-motor integration, eye-hand coordination and quality of handwriting. Journal of Occupational Therapy, Schools \& Early Intervention. 2009; 2(2):87-95. [DOI:10.1080/19411240903146228]

[19] Feder KP, Majnemer A. Handwriting development, competency, and intervention. Developmental Medicine \& Child Neurology. 2007; 49(4):312-7. [DOI:10.1111/j.14698749.2007.00312.x] [PMID] 